\title{
Use of the Cytobrush for concurrent endocervical cytology and chlamydia sampling
}

\author{
Anne Szarewski, Anna Pompey, John Bertrand, Caroline Bradbeer
}

\begin{abstract}
In 899 women attending a genitourinary medicine clinic Chlamydia trachomatis was tested for using both a Cytobrush and a conventional swab. In 425 cases, the Cytobrush was used for concurrent cytology and chlamydia sampling. Both methods were equally effective in the detection of chlamydia, and, in addition, the Cytobrush gave a higher yield of inclusion bodies per sample. The use of the same instrument for both cytology and chlamydia screening may represent a saving in time and money.
\end{abstract}

Chlamydia trachomatis is an important genital pathogen in women. Between $18 \%$ and $31 \%$ of all women attending departments of genitourinary medicine (GUM) have chlamydial infection of the cervix. ${ }^{12} \mathrm{~A}$ study in a family planning clinic showed that $8.2 \%$ of asymptomatic women attending for insertion of an intra uterine contraceptive device and $2.4 \%$ of those attending for routine family planning advice were found to be infected on screening. ${ }^{3}$ Eight per cent of women attending for therapeutic abortion were found to be infected ${ }^{4}$ as well as $8 \%$ of women undergoing vaginal examination in one inner city general practice. 5

It has been estimated that one in ten women who harbour $C$ trachomatis at the cervix develop pelvic inflammatory disease ${ }^{6}$ with its sequelae of infertility and a sevenfold increase in the rate of ectopic pregnancy. $^{7}$ Indeed, in Europe, $60 \%$ of cases of pelvic inflammatory disease may be due to chlamydia, ${ }^{8}$ and similar figures have been quoted for the United States. ${ }^{2}$

Unfortunately, in the majority of cases, uncomplicatecd infection in women is symptomless and may be latent for long periods of time. ${ }^{9}$ Routine screening is therefore important in its detection.

Department of Genitourinary Medicine

Anne Szarewski, Caroline Bradbeer

Department of Virology, St Thomas' Hospital, London UK

Anna Pompey, John Bertrand
$C$ trachomatis is found in endocervical cells. The conventional method of screening has used a cotton tipped swab to sample the endocervix. The Medscand Cytobrush has been shown to be highly effective in sampling endocervical cells. Cervical cytology specimens obtained using the combination of a spatula and a Cytobrush have been shown to contain endocervical cells in $97 \%-98 \%$ of cases. ${ }^{10}{ }^{11}$ In these studies, the use of the combination of a spatula with the Cytobrush resulted not only in better sampling of endocervical cells, but also in a higher yield of positive cervical smear results when compared with the conventional method.

Many patients attending GUM clinics require both cervical cytology and chlamydia screening. In view of the prevalence described above, this may also be the case in other clinics, such as gynaecology and family planning. The purpose of this trial was therefore to ascertain whether the Cytobrush could be used concurrently for endocervical cytological and chlamydial screening, and whether it was then as effective as the conventional swab used for chlamydia screening.

\section{Method}

The patients were unselected women attending the GUM department between September 1988 and January 1989.

The study was performed in two stages. In the first, all patients requiring cervical cytology screening had their smears performed with the combination of an Ayre's spatula followed by a Cytobrush (supplied by Medscand AB Malmo Sweden). The same Cytobrush was then agitated in a container of chlamydial transport medium and discarded. Following this, a second specimen for chlamydia culture was obtained using a wire mounted cotton swab, which was broken off into the transport medium in the usual way.

The order of sampling was reversed in the second stage of the trial. All patients requiring cervical cytology had this performed using an Ayre's spatula only. A specimen for chlamydia culture was then obtained using a cotton tipped wire mounted swab. After this, a second specimen for chlamydia culture was obtained using a Cytobrush.

All the specimens were collected into $2 \mathrm{ml}$ Eagle's 
Table 1 Results of chlamydia culture when the Cytobrush was used first

\begin{tabular}{lllll}
\hline & \multicolumn{5}{l}{ Cytobrush } \\
\cline { 3 - 5 } & & + & - & Total \\
\hline Swab & + & 27 & 4 & 31 \\
& - & 17 & 377 & 394 \\
& Total & 44 & 381 & 425 \\
\hline
\end{tabular}

minimum essential medium and cultured as previously described. ${ }^{12}$ Chlamydiae were identified by finding typical inclusion bodies. Positive samples were graded according to the yield of inclusion bodies.

\section{STATISTICAL METHODS}

Statistical comparisons on the two samples from the same patient were made using the matched pairs test for discordant pairs by means of Fisher's exact test. Comparisons between patients were made by standard chi square methods for $2 \times 2$ tables and a test for trends in proportions. ${ }^{13}$

\section{Results}

During the first stage of the trial (using the Cytobrush first) a total of 850 specimens was obtained from 425 patients, of which 75 specimens $(17.6 \%$ of patients) were positive for chlamydia on culture. In 17 cases the Cytobrush specimen was positive when the swab specimen was negative (table 1). This difference was significant, $p=0.007$.

During the second stage of the trial (using the swab first) a total of 948 specimens was obtained from 474 patients, of which 92 specimens ( $19.4 \%$ of patients) were positive for chlamydia on culture. In six cases the Cytobrush specimen was positive when the swab specimen was negative, while the swab was positive in four cases when the Cytobrush was negative (table 2). This difference was not significant.

These results indicate that the performance of the swab was adversely affected by prior use of the Cytobrush. However, the Cytobrush did not appear to be affected adversely by prior use of the swab. Because of these carry over effects, in subsequent analyses, we have compared the two procedures based on only the first sample. This was done by an unmatched analysis.

When the two methods used first were compared, there was no statistically significant difference between them (table 3). However, a statistically significant difference was found in favour of the Cytobrush with regard to the number of inclusion bodies per sample, $\chi^{2}$ trend $=8 \cdot 12, p=0.005$ (Table 4).
Table 2 Results of chlamydia culture when the swab was used first

\begin{tabular}{llrrr}
\hline & \multicolumn{5}{c}{ Cytoprush } \\
\cline { 3 - 5 } & & + & - & Total \\
\hline Swab & + & 41 & 4 & 45 \\
& - & 6 & 423 & 429 \\
& Total & 47 & 427 & 474 \\
\hline
\end{tabular}

Table 3 Number of positive cultures obtained using each method (first samples only)

\begin{tabular}{ll}
\hline Cytobrush $(n=425)$ & $44(10 \cdot 3 \%)$ \\
Swab $(n=474)$ & $45(9 \cdot 5 \%)$ \\
\hline
\end{tabular}

Table 4 Mean number of inclusion bodies per microscopic field (using $\times 10$ objective), first samples only.

\begin{tabular}{lllll}
\hline & $10-20$ & $20-30$ & $30-40$ & $>40$ \\
\hline Cytobrush & 21 & 6 & 12 & 5 \\
Swab & 33 & 6 & 5 & 1
\end{tabular}

$\chi^{2}$ trend $=8 \cdot 12, p=0.005$.

\section{Discussion}

The Cytobrush has been used successfully for chlamydial sampling in trials in Japan and the USA, ${ }^{14}{ }^{15}$ and indeed has been found to double the detection of chlamydiae when compared with a dacron swab. ${ }^{15}$

Our results indicate that the Cytobrush is an effective chlamydial sampling device, even when it has first been used to take a cervical smear. Indeed, when compared with our current method, it gives a higher yield of inclusion bodies per sample, which may make detection in the laboratory easier. The yield of positive chlamydia results is similar to that previously reported from this and similar clinics. ${ }^{15} 16$

Our finding that the use of the Cytobrush adversely affects subsequent use of a swab for chlamydial sampling suggests that in cases where the Cytobrush is being used for cytological sampling, subsequent use of a swab for chlamydial sampling will yield inferior results. In such instances, the use of the Cytobrush for both procedures is clearly preferable.

Staff in the clinic found the Cytobrush quicker and easier for chlamydia sampling, as the technique for breaking the wire mounted swab is relatively cumbersome.

The cost of a wire mounted cotton tipped swab in our clinic is nearly twice that of a Cytobrush. The concurrent use of the Cytobrush for cervical cytology and chlamydia sampling is efficient and saves time and money. This technique will be valuable in family planning clinics, well women clinics and gyraecology 
departments where chlamydia transport medium is available. It would be worth extending the study by including enzyme assay and immunofluorescence methods.

We thank Dr Jack Cuzick of the Imperial Cancer Research Fund, London, and Ms. Julie Whatley of St Thomas' for their assistance in the statistical analysis of the results.

Cytobrushes and financial assistance for extra chlamydia culture samples were provided by Medscand AB, Malmo, Sweden.

Address for reprints: Anne Szarewski, Lydia Dept. St. Thomas' Hospital, London SE1 7EH UK

\section{References}

1 Schachter J. Medical Progress, Chlamydial Infections. $N$ Engl J Med 1978;298:540-49.

2 Schachter J. Why we need a program for the control of chlamydia trachomatis. N Engl J Med 1989;320:802-4.

3 Fish ANJ, Robinson G, Bounds W, Fairweather DVI, Guillebaud J, Oriel JD, Ridgway GL. Chlamydia trachomatis in various groups of contraceptors: preliminary observations. Br J Family Planning 1987;13:84-7.

4 Ridgway GL, Mumtaz G, Stephens RA, Oriel JD. Therapeutic abortion and chlamydial infection. Br Med $J$ 1983;286: 1478-9.

5 Southgate LJ, Treharne JD, Forsey T. Chlamydia trachomatis and Neisseria gonorrhoeae infections in women attending inner city general practices. $\mathrm{Br} \mathrm{Med} J$ 1983;287:879-81.
6 Mardh PA. Ascending chlamydial infection in the female genital tract. In: Chlamydial Infections. Proceedings of the 6th International Symposium on Human Chlamydial Infections. Oriel JD, Ridgway GL, Schachter J, Taylor-Robinson D, Ward M. Cambridge: Cambridge University Press, 1986.

7 Westrom L. Reproductive events after acute salpingitis. Charing Cross Current Fertility Control Symposium. Br J Clin Pract. Symposium supplement 1982;17:17-20.

8 Westrom L, Mardh PA. Current views on the concept of pelvic inflammatory disease. Aust NZ J Obstet Gynaecol 1984;24: 98-105.

9 McCormack WM, Alpert A, McComb D, et al. Fifteen month follow up study of women infected with $\mathrm{C}$ Trachomatis. $N$ Engl J Med 1979;300:123-5.

10 Boon ME, Alons van Kordelaar JJM, Rietveld-Scheffers PEM. Consequences of the introduction of combined spatula and Cytobrush sampling for cervical cytology: improvements in smear quality and detection rates. Acta Cytol 1986;30:264-70.

11 Taylor PT, Andersen WA, Barber SR, Covell JL, Blair Smith E, Underwood PB. The screening Papanicolaou smear: contribution of the endocervical brush. Obstet Gynecol 1987;70:734-7.

12 Bradbeer CS, Thin RN. Comparison of econazole and isoconazole as a single dose treatment for vaginal candidosis. Genitourin Med 1985;61:396-8.

13 Armitage P, Berry G. Statistical methods in medical research. 2nd Ed. 1987. Oxford: Blackwell Scientific Publications.

14 Kobayashi TK, Ueda M, Araki H, Toyoda K, Ohmori $\mathrm{K}$, Sawaragi I. Immunocytochemical demonstration of chlamydia infection in the urogenital tracts. Diagn Cytopathol 1987;3:303-7.

15 Ciotti RA, Sondheimer SJ. Nachamkin I. Detecting Chalymdia Trachomatis by direct immunofluorescence using a Cytobrush sampling technique. Genitourin Med 1988;64: 245-6.

16 Schachter J, Stoner E, Moncada J. Screening for chlamydial infections in women attending family planning clinics: evaluations of presumptive indicators for therapy. West $J$ Med 1983;138:375-9.

Accepted for publication 28 September 1989 